\title{
Characteristics of Resistance to Puccinia coronata f. sp. avenae in Avena fatua
}

Edyta Paczos-Grzeda, ${ }^{\dagger}$ Sylwia Sowa, and Aneta Koroluk, Institute of Plant Genetics, Breeding and Biotechnology, University of Life Sciences in Lublin, Lublin, Poland; and Tim Langdon, Institute of Biological, Environmental and Rural Sciences, Aberystwyth University, Aberystwyth, UK

\begin{abstract}
Crown rust, caused by Puccinia coronata f. sp. avenae, is the most widespread and harmful fungal disease of oat. The best defense against the pathogen is use of cultivars with genetic resistance, which is effective, economic, and an environmentally friendly alternative to chemical control. However, the continuous evolution of the pathogen can rapidly overcome major gene resistance, creating an urgent need to identify new sources. Wild oat accessions have already proven to be valuable donors of many resistance genes, but the weed species Avena fatua remains underexploited. Its abundance across multiple environments and the frequent occurrence of herbicide-resistant populations demonstrate its ready ability to adapt to biotic and abiotic stresses; yet, surprisingly, there are no extensive studies which describe crown rust resistance occurrence in gene bank stocks of $A$. fatua. In this study, 204 accessions of A. fatua maintained in the collections of the United States Department of Agriculture (USDA) and Polish National Centre for Plant Genetic Resources were evaluated at the seedling stage for

crown rust reaction using host-pathogen tests with five highly diverse and virulent races of $P$. coronata. Of tested genotypes, $85 \%$ showed a heterogeneous infection pattern, while $61 \%$ were susceptible or moderately susceptible to all races. Of the 79 resistant $A$. fatua accessions, seedling resistance to at least two $P$. coronata isolates was recognized within 19 accessions, with 13 displaying a homogeneously resistant phenotype to one or two races. Accessions showing multiple single seedling resistance to three or four isolates were observed. Based on the seedling reaction to isolates used in the study, 18 infection profiles (IP) were determined. Using UPGMA clustering, resistant accessions were divided into six main clusters encompassing samples with similar IPs. Twelve of 18 patterns allowed us to postulate the likely presence of novel crown rust resistance genes, whose origin was predominantly from Kenya or Egypt. Future work will clarify the genetic basis of the resistances observed here, as well as confirm their potential utility in breeding resistant oat cultivars.
\end{abstract}

Crown rust, caused by Puccinia coronata Cda. f. sp. avenae, is considered the most damaging disease of oat, and the use of racespecific $(P c)$ genes for resistance has been the primary means of control (Gnanesh et al. 2014). In the Avena genus, over 100 Pc genes have been discovered (Chong et al. 2011; Gnanesh et al. 2013). Many of these genes originated from hexaploids $A$. sativa L. $(P c 3 c, P c 4 c, P c 6 c, P c 6 d, P c 9, P c 13, P c 22, P c 95, P c 96)$ and A. byzantina Koch. (Pc1-Pc8, Pc9c, Pc10-Pc12, Pc14, Pc21), but the main sources of crown rust resistance were accessions of wild hexaploid A. sterilis L. collected in the 1960s and 1970s from Israel and other Mediterranean countries (Cabral and Park 2014; CDL 2017; Gnanesh et al. 2014; Šebesta et al. 2003; Simons et al. 1978). Diploid and tetraploid Avena species have also been broadly investigated as a potential source of new, effective $P c$ genes. Resistant genotypes were identified in diploids: A. atlantica Baum et Fedak, A. damascena Rajhathy et Baum, A. hirtula Lag., A. longiglumis Dur., A. strigosa Schreb., and A. wiestii Steud, as well as tetraploids: A. agadiriana Baum et Fedak, A. barbata Pott ex Link, $A$. magna Murphy et Terrell, and A. murphyi Ladiz (Aung et al. 2010; Cabral and Park 2014; Cabral et al. 2011; Carson 2009a, 2010; CDL 2017; Mitchell Fetch et al. 2007; Nazareno et al. 2017; Rines et al. 2018; Sowa et al. 2016; Tan and Carson 2013). Resistance appears to be particularly common in the diploid A. strigosa (AsAs)

${ }^{\dagger}$ Corresponding author: E. Paczos-Grzeda;

E-mail: edyta.paczos@up.lublin.pl

Funding: This research was supported by Ministry of Agriculture and Rural Development of the Republic of Poland Grant 2014-2020: "Crown rust resistance genes pyramiding in oat genome and identification of DNA markers for these genes".

*The $\boldsymbol{e}$-Xtra logo stands for "electronic extra" and indicates that one supplementary table is published online.

Accepted for publication 6 June 2018.

() 2018 The American Phytopathological Society
(Pc15, Pc16, Pc17, Pc19, Pc23, Pc30, Pc37, Pc81, Pc82, Pc83, $P c 84, P c 85, P c 86, P c 87, P c 88, P c 89, P c 90, P c 94$ ) (Cabral et al. 2014; CDL 2017) and the tetraploid A. barbata (AABB) (Carson 2009a, 2010). However, all diploid and tetraploid Avena species belong to the tertiary gene pool. Problems arise during introgression of resistance from these species into cultivated hexaploid oat as a result of differences in the ploidy level and reduced chromosome homology, which prevents pairing of homologous chromosomes (Jellen and Leggett 2006). Exploitation of resistance from these species is consequently laborious, and despite the large number of known resistance genes within the tertiary germplasm, only $P c 23$ and $P c 94$ derived from $A$. strigosa have been incorporated into A. sativa, although $P c 94$ has been widely used in resistance breeding (Aung et al. 1996; Dyck and Zillinsky 1963). Despite the large number of resistance genes identified within the tertiary Avena pool, due to barriers in the integration of resistance, $P c$ genes introduced from hexaploid oats: A. sativa, A. byzantina, and A. sterilis, were mainly used in breeding programs in North America, Europe, and Australia (Pc38, Pc39, Pc48, Pc50, Pc58, Pc59, Pc60, Pc61, Pc68) (Cabral and Park 2014; Gnanesh et al. 2014; Šebesta et al. 2003).

The primary gene pool of Avena contains a number of hexaploid species besides the A. byzantina, A. sativa, and A. sterilis mentioned above. These include A. fatua L., A. ludoviciana Durieu, and A. occidentalis Durieu. A. fatua is the most widely distributed of all of these and is considered one of the 10 worst annual weeds of cereal crops globally (Holm et al. 1991; Loskutov and Rines 2011). Its abundance across multiple environments, and the frequent occurrence of herbicide-resistant populations (Adamczewski et al. 2013; Owen and Powles 2009; Thill et al. 1994), demonstrate its ready ability to adapt to biotic and abiotic stresses; yet, surprisingly, no extensive studies have been reported of A. fatua potential as a source of crown rust resistance. The large collections of $A$. fatua held in gene banks across the world, running to thousands of accessions, could be a very rich resource, which could be easily deployed in breeding for crown rust resistance. The objective of this research was to identify and characterize resistance to $P$. coronata within a representative selection of $A$. fatua accessions maintained in United States Department 


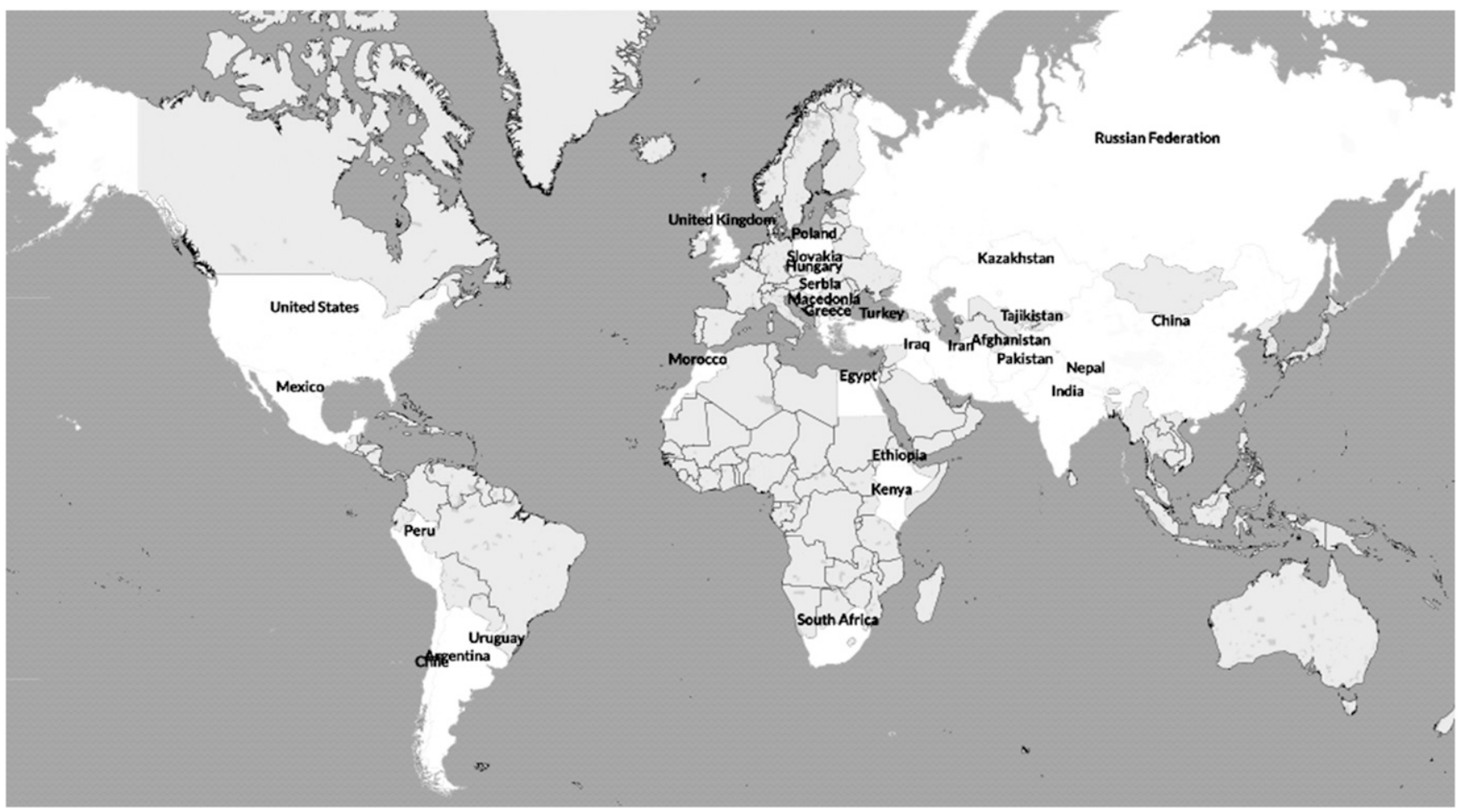

Fig. 1. Distribution of Avena fatua genotypes used in the study for searching new sources of resistance to crown rust in oat.

of Agriculture (USDA) and Polish National Centre for Plant Genetic Resources collections.

\section{Materials and Methods}

A set of 204 accessions of A. fatua from different parts of the world was chosen proportionally to the availability of accessions in the gene banks. Examined plant material came originally from 29 countries located in North and South America, Asia, Europe, and Africa (Fig. 1, Table 1, Supplementary Table S1). Seeds of A. fatua accessions were obtained from the USDA National Small Grains Collection Aberdeen, Idaho, USA (186 accessions) and the National Centre for Plant Genetic Resources, Radzików, Poland (18 accessions). Crown rust resistance of each of the genotypes was tested using five highly virulent $P$. coronata isolates (Pca) by means of host-pathogen test (Hsam et al. 1997). Crown rust pathogen races CR230, CR241, and CR257 were kindly supplied by Dr. J. Menzies from Morden Research and Development Centre, AAFC, Canada along with their virulence characterization in relation to $18 P c$ genes (Table 2). Pca isolates $94 \mathrm{U}(63)$ and $51 \mathrm{U}(22)$ were selected from a wide collection of single-pustule pathotypes derived from populations collected in Poland in the years 2010-2014 according to the method described by Sowa et al. (2016).

Before the main experiment, the virulence of each isolate was tested on a set of 34 differential oat lines with single resistance genes as well as control cultivar Kasztan. Differentials for genes $P c 58$, $P c 59, P c 60$, and Pc61 were represented by cultivars TAM-O-301, TAM-O-312, Coker227, and Coker234, respectively (Simons et al. 1978). The near-isogenic lines with genes Pc35, Pc38, Pc39, Pc40, Pc45, Pc46, Pc48, Pc50, Pc54, Pc55, Pc56, Pc57, Pc62, Pc63, Pc64, Pc68, Pc91, Pc94, Pc96, Pc97, Pc98, Pc101, Pc1031, Pc104 were developed at the Cereal Research Centre AAFC, Winnipeg, Canada; and the differentials representing Pc14, Pc36, Pc51, $P c 52, P c 70, P c 71$ genes were created at Iowa State University (Carson 2011; Chong et al. 2011; Menzies et al. 2015). For subsequent inoculation, microfuge tubes filled with urediniospores were heat-shocked for $4 \mathrm{~min}$ at $42^{\circ} \mathrm{C}$ and multiplied on leaf fragments of 10-day-old seedlings of susceptible cultivar Kasztan using the host-pathogen test procedure. Leaves were placed into petri dishes filled with agar $(0.6 \%)$ with the addition of benzimidazole
(3.4 $\mathrm{mM})$ and inoculated in a settling tower by applying 500-700 spores of $P$. coronata per square centimeter. The dishes were incubated for 10 days in a phytotron at about $18^{\circ} \mathrm{C}$ with $70 \%$ humidity and light intensity of approximately $4 \mathrm{kLx}$. In order to define virulence of the fungal samples, each of the isolates was examined on a set of reference oat lines. Seeds of $P c$ lines were placed on plug trays filled with a universal substrate mixed with peat. Seedlings were grown for 10 days and used to perform host-pathogen tests according to the methodology described above. Leaves were placed onto 12-well culture plates filled with agar using Kasztan as an infection control in each well. Disease symptoms were assessed on three leaf fragments, each from a different seedling of one reference oat line. A crown rust reaction of medium-sized to large colonies with little or no chlorosis was considered to be susceptible, whereas lines with no visible symptoms as well as small or no colonies with necrotic and chlorotic changes were scored as resistant.

To screen the susceptibility of the gene bank accessions, A. fatua grains were seeded onto plug trays filled with a universal substrate with peat, and allowed to germinate. After 10 days, six leaf fragments, each from a different seedling of the $A$. fatua accessions, were placed on 12-well culture plates with agar and benzimidazole medium. Control cultivar leaf was put into each well of a plate. The plates were inoculated with the selected $P$. coronata isolates as detailed and incubated in a growth chamber for 10 days according to the methodology described above. Crown rust disease symptoms were evaluated using the qualitative scale of S, MS, MR, R, and $\mathrm{HR}$, where $\mathrm{S}=$ susceptible - large to moderately large pustules with little or no chlorosis; MS = moderately susceptible - moderately large pustules surrounded by extensive chlorosis; MR = moderately resistant - small pustule surrounded by chlorosis; $\mathrm{R}=$ resistant chlorotic or necrotic flecking; and $0=\mathrm{HR}=$ highly resistant - no visible reaction (Carson 2009a; Sowa et al. 2016).

Reactions to the isolate infections were grouped into two classes: phenotypes described as S and MS were considered as susceptible, and the remainder as resistant. In order to select genotypes with crown rust resistance conditioned by genes not described so far, the infection profiles (IP) of differential lines were compared with the infection profiles assigned to analyzed $A$. fatua accessions. Scores of infection were transformed into a binary matrix to perform 
hierarchical analysis. Susceptibility or resistance to each $P$. coronata pathotype was considered as 1 and 0 , respectively. Accessions with uniform susceptibility reaction were excluded from the analysis, remaining data were used to conduct clustering and construct a similarity dendrogram based on the unweighted pair-group method (UPGMA) in PAST 3.19 software (Hammer et al. 2001). Groups and subgroups were determined using Dice similarity coefficients (Dice 1945) with 1,000 bootstraps.

\section{Results}

The $P$. coronata isolates used in this research were selected to overcome immunity of resistance genes in the selected reference lines and thus exclude the presence of these genes in screened genotypes. The most aggressive, inducing susceptible response in 18 of 34 evaluated $P c$ genes, was pathotype $94 \mathrm{U}(63)$. CR230 and 51U(22) isolates overcame the resistance of 15 reference lines, whereas CR241 and CR257 were virulent toward 13 and $11 P c$ lines, respectively (Table 2).

All $P$. coronata isolates were used to perform the host-pathogen tests on the $204 \mathrm{~A}$. fatua accessions. Resistance reactions ranged from susceptible (S) to highly resistant (HR) (Table 1). Most of the tested A. fatua accessions $(173 ; 85 \%)$ displayed heterogeneous phenotypes, and the response of single seedlings within accessions to specific Pca inoculation could vary even from susceptible to highly resistant. Nevertheless, 125 accessions were completely susceptible for all tested races of $P$. coronata. For 33 accessions, only single seedlings were rated as resistant or moderately resistant to one crown rust race, whereas in 20 accessions more than one of six screened seedlings displayed a variable level of resistance to one or two Pca pathotypes, most of which were resistant to 51(22), CR257, or CR230 (Table 1). For 13 accessions, more than one seedling was resistant, but each plant responded to one to three different Pca pathotypes. The remaining 13 accessions (6.4\%) displayed a consistent resistant phenotype. Four of these accessions were immune to race CR257 (PI 411458, PI 411491) or CR230 (PI 362374, PI 411476), and eight were assessed as resistant simultaneously to 94U(63) and CR257 strains. Distinct, but also homogenous and comparably effective patterns of resistance on races CR230 and 51(22) were presented by the Egyptian PI365611 accession. In some of the accessions described above, multiple resistance of single seedlings was observed. Single plants of PL 502694, PI 411458, PI 411465, and PI 411469 were resistant to three $P$. coronata isolates, and two seedlings of PI 365611 from Egypt showed resistance to four isolates.

Table 1. Characteristics of resistant Avena fatua accessions reactions on inoculation with 5 Puccinia coronata isolates

\begin{tabular}{|c|c|c|c|c|c|c|c|c|}
\hline \multirow[b]{2}{*}{ Plant ID } & \multirow[b]{2}{*}{ GenBank $^{\mathbf{b}}$} & \multirow[b]{2}{*}{ Origin } & \multicolumn{5}{|c|}{$P$. coronata race $^{\mathrm{a}}$} & \multirow{2}{*}{$\begin{array}{l}\text { Infection profile (number } \\
\text { of resistant seedlings) }\end{array}$} \\
\hline & & & $51(22)$ & $94(63)$ & CR230 & CR241 & CR257 & \\
\hline PI 269358 & NSGC & Afghanistan, Kandahar & S,MS,MR & $\mathrm{S}$ & S,MS & S,MS & S,MS & $1(1)$ \\
\hline PI 269870 & NSGC & Pakistan, North-West Frontier & MS,MR & S,MS & $\mathrm{S}$ & $\mathrm{S}$ & MS & $1(1)$ \\
\hline PI 287310 & NSGC & Greece, Peloponnese & MS,R & MS & MS & MS & MS & $1(1)$ \\
\hline PI 365602 & NSGC & Iraq & MS,R & S,MS & MS & $\mathrm{S}$ & MS & $1(1)$ \\
\hline PI 365603 & NSGC & Iraq & S,MS,R & MS & MS & $\mathrm{S}, \mathrm{MS}$ & S,MS & $1(1)$ \\
\hline PI 432465 & NSGC & United States, Montana & S,MS,R & S,MS & MS & MS & MS & $1(1)$ \\
\hline PI 538801 & NSGC & Russian Federation, Altay & MS,R & MS & MS & MS & $\mathrm{S}$ & $1(1)$ \\
\hline PI 545392 & NSGC & United States, Utah & MS,R & $\mathrm{S}$ & $\mathrm{S}$ & $\mathrm{S}$ & $\mathrm{S}$ & $1(1)$ \\
\hline PI 545393 & NSGC & United States, Utah & MS,R & $\mathrm{S}$ & $\mathrm{S}$ & $\mathrm{S}$ & $\mathrm{S}$ & $1(1)$ \\
\hline PL 51532 & IHAR & Peru & S,MS,R & $\mathrm{S}$ & MS & $\mathrm{S}$ & $\mathrm{S}$ & $1(1)$ \\
\hline PL 51702 & IHAR & Poland & S,MS,R & S,MS & MS & $\mathrm{S}$ & MS & $1(1)$ \\
\hline PI 287309 & NSGC & Greece, Peloponnese & MS,MR & MS & MS & MS & MS & $1(1)$ \\
\hline PI 366424 & NSGC & Afghanistan, Parwan & MS,R & MS & $\mathrm{S}$ & MS & $\mathrm{S}$ & $1(2)$ \\
\hline PI 374393 & NSGC & Serbia & MS,R & MS & S,MS & S,MS & $\mathrm{S}$ & $1(2)$ \\
\hline PI 545394 & NSGC & United States, Utah & MS,R & $\mathrm{S}$ & $\mathrm{S}$ & $\mathrm{S}$ & S,MS & $1(2)$ \\
\hline PI 388760 & NSGC & Morocco, Taroudannt & MS,R & S,MS & $\mathrm{S}, \mathrm{MS}$ & $\mathrm{S}$ & $\mathrm{S}$ & $1(3)$ \\
\hline PI 411494 & NSGC & Turkey, Erzurum & MS,R & MS & MS & MS & $\mathrm{S}$ & $1(3)$ \\
\hline PI 210998 & NSGC & Afghanistan, Bamian & $\mathrm{S}, \mathrm{MS}$ & S,MS,R & $\mathrm{S}$ & $\mathrm{S}$ & S,MS & $2(1)$ \\
\hline PI 545371 & NSGC & United States, Minnesota & $\mathrm{S}, \mathrm{MS}$ & MS,MR & S,MS & $\mathrm{S}$ & $\mathrm{S}$ & $2(1)$ \\
\hline PI 545377 & NSGC & United States, Montana & S,MS & MS,MR & S,MS & $\mathrm{S}$ & $\mathrm{S}$ & $2(2)$ \\
\hline PI 228338 & NSGC & Iran, East Azerbaijan & $\mathrm{S}, \mathrm{MS}$ & MS & MS,R & MS & MS & $3(1)$ \\
\hline CIav 2526 & NSGC & United Kingdom, Wales & S,MS & $\mathrm{S}$ & MS,MR & MS & MS & $3(1)$ \\
\hline PI 269360 & NSGC & Afghanistan, Bamian & MS & S,MS & S,MS,MR & S,MS & MS & $3(1)$ \\
\hline PI 344854 & NSGC & Serbia & S,MS & S,MS & MS,R & S,MS & MS & $3(1)$ \\
\hline PI 544414 & NSGC & United States, Idaho & MS & MS & MS,MR & MS & S,MS & $3(1)$ \\
\hline CIav 3213 & NSGC & United States, New York & S,MS & $\mathrm{S}, \mathrm{MS}$ & MS,MR & $\mathrm{S}$ & $\mathrm{S}$ & $3(1)$ \\
\hline PI 287312 & NSGC & Greece & MS & S,MS & MS,R & S,MS & MS & $3(2)$ \\
\hline PI 639335 & NSGC & Tajikistan, Badakhshoni Kuhi & $\mathrm{S}$ & $\mathrm{S}$ & S,MS,MR & S,MS & $\mathrm{S}$ & $3(2)$ \\
\hline PI 411470 & NSGC & Turkey, Erzurum & $\mathrm{S}$ & MS & MS,MR,R & MS & $\mathrm{S}$ & $3(3)$ \\
\hline PI 165601 & NSGC & India, Uttar Pradesh & S,MS & MS & MS,R & S,MS & S,MS & $3(4)$ \\
\hline PI 365600 & NSGC & Iraq & $\mathrm{S}$ & S,MS & MS,R & MS & MS & $3(5)$ \\
\hline PI 411476 & NSGC & Turkey, Van & $\mathrm{S}$ & $\mathrm{S}$ & $\mathrm{R}$ & MS & MS & $3(6)$ \\
\hline PI 266828 & NSGC & United Kingdom, England & MS & S,MS & MS & S,MR & MS & $4(1)$ \\
\hline PI 447299 & NSGC & China, Gansu & MS & MS & MS & MS,R & $\mathrm{S}$ & $4(1)$ \\
\hline PI 544372 & NSGC & United States, North Dakota & MS & MS & MS & MS,R & S,MS & $4(1)$ \\
\hline PI 544697 & NSGC & United States, Wyoming & MS & MS & S,MS & MS,R & MS & $4(1)$ \\
\hline \multirow[t]{2}{*}{ PI 545352} & NSGC & United States, Arizona & MS & S,MS & MS & MS,MR & S,MS & $4(1)$ \\
\hline & & & & & & & & (Continued on next page) \\
\hline
\end{tabular}

\footnotetext{
${ }^{a}$ Resistance phenotype: $\mathrm{S}=$ susceptible, large to moderately large pustules with little or no chlorosis; MS = moderately susceptible, moderately large pustules surrounded by extensive chlorosis; $\mathrm{MR}=$ moderately resistant, small pustule surrounded by chlorosis; $\mathrm{R}=$ resistant, chlorotic or necrotic flecking; and $\mathrm{HR}=$ highly resistant, no visible reaction.

b IHAR - National Centre For Plant Genetic Resources, The Plant Breeding and Acclimatization Institute - NRI, Radzików, Poland; NSGC - National Small Grains Collection, Aberdeen, Idaho, USA.

${ }^{\mathrm{c}} \mathrm{IP}$ - infection profile - infection pattern determined for five tested isolates; number of resistant seedlings in brackets.
} 
Based on the seedling reactions to the five $P$. coronata isolate inoculations, 18 infection profiles (IPs) of screened $A$. fatua accessions were determined (Table 3 ). IP 0 corresponded to highly virulent reactions to all of the crown rust races. Resistance to one of five pathotypes was assigned as IP 1 - IP 5, while resistance to two $P$. coronata isolates was described as IP $6-$ IP 11 . IP $12-16$ indicated a combination of resistance to three crown rust races. Resistance to four pathotypes: 51(22), CR230, CR241, CR257, was reported only once (PI 365611 Minya, Egypt), and was assigned as IP 17 (Table 1). Next, the infection profiles of the A. fatua accessions were compared with IPs of differential $P c$ lines. The infection pattern of reference lines containing genes $P c 36, P c 39, P c 55, P c 61, P c 70$, and $P c 71$ were identical to IP 1. Pc38 and Pc63 lines corresponded to IP 6, as observed for PI 221435 (Afghanistan) and PI 411453 (Iran). IP 10 was one of the most frequently appearing profiles, characterizing almost all accessions from Kenya as well as two accessions from the United States (PI 545361, PI 545479). This infection scheme fitted the phenotype of the oat differential line containing Pc14. Resistance to 51(22), CR230, and CR240 was characteristic of the differential line for $P c 94$. This pattern was assigned as IP 13 and observed for accession PI 365611 from Egypt. IP 14 was observed for lines Pc48, Pc103-1, and accession 502694 from Iran. IP 16 corresponded to resistance to crown rust isolates 94(63), CR241, CR257, and was seen in seedlings of two accessions from the Rift Valley in Kenya (PI 411458, PI 411465) as well as the oat differential line for Pc35.

The virulence level of the crown rust isolates used in the study was very high and relatively similar, ranging from 84 to $93 \%$, taking into account single seedling response (Table 1). Pathotypes CR241 and 94(63) were found to be the most virulent, infecting single seedlings of 190 and 186 accessions, respectively. No cases of consistent resistance to isolate CR241 were reported. Out of 204 analyzed accessions, only eight displayed simultaneous homogenous resistance to isolates 94U(63) and CR257, whereas only one equal and highly resistant reaction to both isolates $51 \mathrm{U}(22)$ and CR230 was ascertained.

Using UPGMA clustering based on the Dice coefficient calculated on infection data, we separated the 79 accessions which were characterized by at least moderate resistance of single seedling to crown rust into six main divisions (Fig. 2). The first and the sixth divisions were represented by single accessions, each characterized by a distinctive and complex resistance profile. All six seedlings of the Egyptian

Table 1. (Continued from previous page)

\begin{tabular}{|c|c|c|c|c|c|c|c|c|}
\hline \multirow[b]{2}{*}{ Plant ID } & \multirow[b]{2}{*}{ GenBank $^{\mathbf{b}}$} & \multirow[b]{2}{*}{ Origin } & \multicolumn{5}{|c|}{$P$. coronata race ${ }^{\mathbf{a}}$} & \multirow{2}{*}{$\begin{array}{l}\text { Infection profile (number } \\
\text { of resistant seedlings) }\end{array}$} \\
\hline & & & $51(22)$ & $94(63)$ & CR230 & CR241 & CR257 & \\
\hline PI 544373 & NSGC & United States, North Dakota & $S$ & MS & $\mathrm{S}, \mathrm{MS}$ & MS,MR & MS & $4(2)$ \\
\hline PI 227353 & NSGC & Iran, Esfahan & S,MS & S & $S$ & $\mathrm{~S}$ & S,MS,R & $5(1)$ \\
\hline PI 344859 & NSGC & Macedonia & MS & $\mathrm{S}$ & MS & $S$ & MS,MR & $5(1)$ \\
\hline PI 411440 & NSGC & Ethiopia, Gonder & $\mathrm{S}$ & S & MS & MS & MS,R & $5(1)$ \\
\hline PI 411462 & NSGC & Kenya, Rift Valley & S,MS & $\mathrm{S}$ & MS & S,MS & MS,MR & $5(1)$ \\
\hline PI 542090 & NSGC & Nepal & $\mathrm{S}$ & MS & MS & S,MS & S,MS,R & $5(1)$ \\
\hline PL 51633 & IHAR & Poland & $\mathrm{S}$ & MS & S,MS & MS & $\mathrm{MS}, \mathrm{R}$ & $5(1)$ \\
\hline PL 52107 & IHAR & Poland & $S$ & S,MS & $S$ & $\mathrm{~S}$ & S,MS,R & $5(1)$ \\
\hline PL 52366 & IHAR & Poland & S,MS & $\mathrm{S}$ & S,MS & S,MS & S,MS,R & $5(1)$ \\
\hline PI 432464 & NSGC & United States, Montana & S,MS & MS & MS & MS & MS,MR,R & $2(2)$ \\
\hline PI 411454 & NSGC & Iran, East Azerbaijan & S,MS & $\mathrm{S}$ & S,MS & MS & $\mathrm{S}, \mathrm{MS}, \mathrm{R}$ & $5(3)$ \\
\hline PI 411483 & NSGC & Turkey, Kars & $\mathrm{S}$ & S,MS & S,MS & $\mathrm{S}$ & MS,MR & $5(4)$ \\
\hline PI 411491 & NSGC & Turkey, Kayseri & $S$ & MS & MS & $S$ & $\mathrm{R}$ & $5(4)$ \\
\hline PI 365601 & NSGC & Iraq & MS & S,MS & MS & $S$ & MS,MR,R & $5(5)$ \\
\hline PI 411484 & NSGC & Turkey, Kars & $S$ & S,MS & MS & $S$ & $\mathrm{~S}, \mathrm{MR}$ & $5(5)$ \\
\hline PI 436009 & NSGC & Chile, La Araucania & MS & S,MS & S,MS & $\mathrm{S}$ & MS,MR,R & $5(5)$ \\
\hline PI 221435 & NSGC & Afghanistan, Bamian & MS,R & S,MR & MS & S,MS & S,MS & $6(1)$ \\
\hline PI 411453 & NSGC & Iran, East Azerbaijan & $\mathrm{S}$ & $\mathrm{S}, \mathrm{R}$ & MS & MS & MS,R & $6(1)$ \\
\hline PI 411460 & NSGC & Kenya, Rift Valley & S,MS & $\mathrm{R}$ & MS & MS & $\mathrm{R}$ & $10(6)$ \\
\hline PI 411463 & NSGC & Kenya, Rift Valley & S,MS & $\mathrm{R}$ & MS & MS & HR & $10(6)$ \\
\hline PI 411466 & NSGC & Kenya, Rift Valley & S,MS & $\mathrm{R}$ & MS & S,MS & HR & $10(6)$ \\
\hline PI 411468 & NSGC & Kenya, Central & MS & $\mathrm{R}$ & MS & MS & HR & $10(6)$ \\
\hline PI 545361 & NSGC & United States, Texas & $S$ & HR & S,MS & $\mathrm{S}$ & HR & $10(6)$ \\
\hline PI 545479 & NSGC & United States, Oklahoma & $S$ & $\mathrm{R}$ & S,MS & $\mathrm{S}$ & HR & $10(6)$ \\
\hline PI 365608 & NSGC & Egypt, Daqahliya & $\mathrm{MS}, \mathrm{MR}, \mathrm{R}$ & $\mathrm{S}, \mathrm{MS}, \mathrm{R}$ & S,MS & MS & MS & $1(2) ; 2(1)$ \\
\hline CIav 4644 & NSGC & Argentina & MS, MR & S,MS & MS,R & $\mathrm{S}$ & MS & $1(1) ; 3(1)$ \\
\hline PI 344855 & NSGC & Serbia & MS,R & MS & S,MS,MR & S & MS & $1(1) ; 3(1)$ \\
\hline PI 344857 & NSGC & Serbia & S,MS,MR & MS & MS,MR & S,MS & S,MS & $1(1) ; 3(1)$ \\
\hline PI 436023 & NSGC & Chile, Los Lagos & MS,R & MS & MS & MS,MR,R & S,MS & $1(1) ; 4(3)$ \\
\hline PI 151083 & NSGC & Uruguay & $S$ & $\mathrm{~S}, \mathrm{MS}, \mathrm{R}$ & $\mathrm{MS}, \mathrm{MR}, \mathrm{R}$ & S,MS & MS & $2(1) ; 3(2)$ \\
\hline PI 349888 & NSGC & Serbia & MS & S,MS & MS,R & $\mathrm{S}$ & $\mathrm{MS}, \mathrm{R}$ & $3(1) ; 5(1)$ \\
\hline CIav 4643 & NSGC & Argentina & MS,S & MS,MR & $\mathrm{MR}, \mathrm{R}$ & $S$ & MS & $3(4) ; 9(2)$ \\
\hline PI 362374 & NSGC & Serbia & $S$ & $\mathrm{~S}$ & $\mathrm{MR}, \mathrm{R}$ & $S$ & S,MS,R & $3(5) ; 11(1)$ \\
\hline PI 411464 & NSGC & Kenya, Rift Valley & S,MS,R & S,MS & MS & MS,MR & $\mathrm{S}, \mathrm{MS}, \mathrm{R}$ & $1(1) ; 4(1) ; 8$ (1) \\
\hline PL 502694 & IHAR & Iran & MS,MR,R & S,MS & $\mathrm{S}, \mathrm{MS}, \mathrm{R}, \mathrm{HR}$ & S,MS,R & S,MS,R & $1(1) ; 4(1) ; 7(1) ; 14(1)$ \\
\hline PI 411485 & NSGC & Turkey, Kars & $\mathrm{S}, \mathrm{MS}, \mathrm{R}$ & MS & MS & MS,MR,R & MS,R & $4(2) ; 8(1)$ \\
\hline PI 411461 & NSGC & Kenya, Rift Valley & S,MS & MS,R & S,MS & MS,MR & MS,R & $4(1) ; 10(5)$ \\
\hline PI 411459 & NSGC & Kenya, Rift Valley & $\mathrm{S}$ & S,MS,R & S,MS & S,MS & $\mathrm{MS}, \mathrm{R}$ & $5(1) ; 10(3)$ \\
\hline PI 411458 & NSGC & Kenya, Rift Valley & S,MS & MS,R & MS & MS,R & MR,R & $5(2) ; 10(2) ; 16(2)$ \\
\hline PI 365611 & NSGC & Egypt, Minya & $\mathrm{R}$ & MS & $\mathrm{R}$ & S,MS,R & MS,R & 7 (2); $13(3) ; 17$ (2) \\
\hline PI 411465 & NSGC & Kenya, Rift Valley & MS & $\mathrm{R}$ & MS & MS,MR & $\mathrm{R}$ & $10(2) ; 16(4)$ \\
\hline PI 411469 & NSGC & Kenya, Central & MS,R & $\mathrm{R}$ & MS,MR & MS & HR & $10(3) ; 12(1) ; 15(2)$ \\
\hline
\end{tabular}


accession PI 365611 belonging to the first cluster displayed resistance to two to four $P$. coronata pathotypes (51U(22), CR230, CR241, and CR257). Resistance was seen in four of six seedlings from the Iranian accession 502694, from the sixth division; each seedling showed a different IP. The second division was composed of nine accessions from Kenya and two from the United States, mostly of IP 10, with crown rust resistance against $94 \mathrm{U}(63)$ and $\mathrm{CR} 257$ races. The fourth cluster contained six accessions of IP 5, resistant toward CR257, whereas the fifth one encompassed four accessions mostly of IP 3 with resistance to CR230 race. The third cluster contained the 56 remaining $A$. fatua accessions, which displayed a relatively low level of resistance to the crown rust isolates used in this study.

With regard to the geographical origin of analyzed materials, A. fatua accessions from Egypt and Kenya exhibited a higher percentage of crown rust resistant seedlings within each genotype in comparison with other countries (Fig. 3). About 15\% of accessions from Argentina, Chile, and Turkey as well as $10 \%$ from Morocco, Uruguay, Iraq, India, Serbia, Iran, and the United States were assessed as fully or partially resistant to a particular Pca race. No resistant forms were identified within A. fatua collected in Mexico or Kazakhstan. The single accessions from Hungary, Slovakia, and South Africa were also crown rust susceptible.

\section{Discussion}

Studies conducted worldwide confirm the extremely virulent dynamics and phenotypic diversity of $P$. coronata $\mathrm{f}$. sp. avenae as a causative agent of crown rust in oats (Carson 2009b, 2011; Chong et al. 2011; Jiráková and Hanzalová 2008; Menzies et al. 2015). The emergence of new rust pathotypes differing in virulence and aggressiveness is linked to the life cycle of the pathogen, involving sexual and asexual reproduction. In the absence of an alternate host on which the sexual part of the cycle and meiotic recombination events take part, new pathotypes arise by mutation and somatic recombination (Chaves et al. 2008; Fetch et al. 2011). Due to the continuous evolution of the pathogen, current sources of resistance to crown rust rapidly lose their effectiveness, so that new ones are urgently needed. Wild oat genotypes have already been proven to be donors of many resistance genes (Gnanesh et al. 2014; Loskutov and Rines 2011); however, A. fatua still remains unexploited.

Previous studies of crown rust resistance genes in A. fatua were limited in terms of number or geographical origin of tested materials. Briggle (1974) identified resistant genotypes in a small collection of A. fatua from California after a test performed with one $\mathrm{Pc}$ race. Simons and Briggle (1984) screened about 400 U.S. accessions of $A$.

Table 2. Virulence profile of Puccinia coronata f. sp. avenae isolates used for testing resistance of Avena fatua accessions

\begin{tabular}{|c|c|c|c|c|}
\hline Isolate number & Phenotype code ${ }^{a}$ & $\begin{array}{l}\text { Virulence to standard } \\
\text { differentials }\end{array}$ & $\begin{array}{c}\text { Virulence to supplemental } \\
\text { differentials } \\
\end{array}$ & Avirulence to differentials \\
\hline $51 \mathrm{U}(22)$ & SBLP & $\begin{array}{l}\text { Pc40, Pc45, Pc46, Pc51, } \\
\text { Pc54, Pc62, Pc64 }\end{array}$ & $\begin{array}{l}\text { Pc14, Pc35, Pc57, Pc96, Pc97, Pc98, } \\
\text { Pc101, Pc104 }\end{array}$ & $\begin{array}{l}\text { Pc36, Pc38, Pc39, Pc48, Pc50, Pc52, Pc56, } \\
\text { Pc58, Pc59, Pc60, Pc61, Pc63, Pc68, Pc70, } \\
\text { Pc71, Pc91, Pc94, Pc103-1 }\end{array}$ \\
\hline $94 \mathrm{U}(63)$ & NJBP & $\begin{array}{l}\text { Pc39, Pc40, Pc46, Pc48, } \\
\quad \text { Pc54, Pc62, Pc64 }\end{array}$ & $\begin{array}{c}\text { Pc36, Pc55, Pc57, Pc61, Pc70, Pc71, } \\
\text { Pc94, Pc96, Рc97, Pc98, Pc103-1 }\end{array}$ & $\begin{array}{l}\text { Pc14, Pc35, Pc38, Pc45, Pc50, Pc51, Pc52, } \\
\text { Pc56, Pc58, Pc59, Pc60, Pc63, Pc68, Pc91, } \\
\text { Pc101, Pc104 }\end{array}$ \\
\hline CR230 & LQCB & Pc38, Pc39, Pc40, Pc59 & $\begin{array}{l}\text { Pc14, Pc35, Pc36, Pc55, Pc57, Pc60, } \\
\text { Pc61, Pc63, Pc70, Pc71, Pc91 }\end{array}$ & $\begin{array}{l}\text { Pc45, Pc46, Pc48, Pc50, Pc51, Pc52, Pc54, } \\
\text { Pc56, Pc58, Pc62, Pc64, Pc68, Pc } 94, \text { Pc } 96, \\
\text { Pc97, Pc98, Pc101, Pc103-1, Pc104 }\end{array}$ \\
\hline CR241 & DSGB & Pc38, Pc39, Pc46, Pc48, Pc52 & $\begin{array}{l}\text { Pc14, Pc36, Pc55, Pc61, Pc63, Pc70, } \\
\quad \text { Pc71, Pc103-1 }\end{array}$ & $\begin{array}{l}P c 35, P c 40, P c 45, P c 50, P c 51, P c 54, P c 56 \\
\quad P c 57, P c 58, P c 59, P c 60, P c 62, P c 64, P c 68 \\
P c 91, P c 94, P c 96, P c 97, P c 98, P c 101, \\
P c 104\end{array}$ \\
\hline CR257 & BRBG & Pc38, Pc39, Pc56, Рc68 & $\begin{array}{l}\text { Pc36, Pc55, Pc61, Pc63, Pc70, Pc71, } \\
\quad \text { Pc94 }\end{array}$ & $\begin{array}{l}P c 14, P c 35, P c 40, P c 45, P c 46, P c 48, P c 50 \\
P c 51, P c 52, P c 54, P c 57, P c 58, P c 59, P c 60 \\
P c 62, P c 64, P c 91, P c 96, P c 97, P c 98, \\
P c 101, P c 103-1, P c 104\end{array}$ \\
\hline
\end{tabular}

a Phenotype code based on the standard differentials set Chong et al. (2000).

${ }^{b}$ Chong et al. (2000).

${ }^{c}$ Chong et al. (2011).

Table 3. Infection profiles (IP) of screened Avena fatua accessions based on the reaction to Puccinia coronata race infection

\begin{tabular}{|c|c|c|c|c|c|c|}
\hline \multirow[b]{2}{*}{ IP } & \multicolumn{5}{|c|}{ P. coronata race } & \multirow{2}{*}{$\begin{array}{l}\text { Oat differential line } \\
\text { with a corresponding phenotype }\end{array}$} \\
\hline & $51(22)$ & $94(63)$ & CR230 & CR241 & CR257 & \\
\hline 0 & $\mathrm{H}^{\mathrm{a}}$ & $\mathrm{H}$ & $\mathrm{H}$ & $\mathrm{H}$ & $\mathrm{H}$ & - \\
\hline 1 & $\mathrm{~L}$ & $\mathrm{H}$ & $\mathrm{H}$ & $\mathrm{H}$ & $\mathrm{H}$ & Pc36, Pc39, Pc55, Рc61, Pc70, Pc71 \\
\hline 2 & $\mathrm{H}$ & $\mathrm{L}$ & $\mathrm{H}$ & $\mathrm{H}$ & $\mathrm{H}$ & - \\
\hline 3 & $\mathrm{H}$ & $\mathrm{H}$ & $\mathrm{L}$ & $\mathrm{H}$ & $\mathrm{H}$ & - \\
\hline 4 & $\mathrm{H}$ & $\mathrm{H}$ & $\mathrm{H}$ & $\mathrm{L}$ & $\mathrm{H}$ & - \\
\hline 5 & $\mathrm{H}$ & $\mathrm{H}$ & $\mathrm{H}$ & $\mathrm{H}$ & $\mathrm{L}$ & - \\
\hline 6 & $\mathrm{~L}$ & $\mathrm{~L}$ & $\mathrm{H}$ & $\mathrm{H}$ & $\mathrm{H}$ & Pc38, Pc63 \\
\hline 7 & $\mathrm{~L}$ & $\mathrm{H}$ & $\mathrm{L}$ & $\mathrm{H}$ & $\mathrm{H}$ & - \\
\hline 8 & $\mathrm{~L}$ & $\mathrm{H}$ & $\mathrm{H}$ & $\mathrm{L}$ & $\mathrm{H}$ & - \\
\hline 9 & $\mathrm{H}$ & $\mathrm{L}$ & $\mathrm{L}$ & $\mathrm{H}$ & $\mathrm{H}$ & - \\
\hline 10 & $\mathrm{H}$ & $\mathrm{L}$ & $\mathrm{H}$ & $\mathrm{H}$ & $\mathrm{L}$ & Pc14 \\
\hline 11 & $\mathrm{H}$ & $\mathrm{H}$ & $\mathrm{L}$ & $\mathrm{H}$ & $\mathrm{L}$ & - \\
\hline 12 & $\mathrm{~L}$ & $\mathrm{~L}$ & $\mathrm{H}$ & $\mathrm{H}$ & $\mathrm{L}$ & - \\
\hline 13 & $\mathrm{~L}$ & $\mathrm{H}$ & $\mathrm{L}$ & $\mathrm{L}$ & $\mathrm{H}$ & Pc94 \\
\hline 14 & $\mathrm{~L}$ & $\mathrm{H}$ & $\mathrm{L}$ & $\mathrm{H}$ & $\mathrm{L}$ & Pc48, Pc103-1 \\
\hline 15 & $\mathrm{H}$ & $\mathrm{L}$ & $\mathrm{L}$ & $\mathrm{H}$ & $\mathrm{L}$ & - \\
\hline 16 & $\mathrm{H}$ & $\mathrm{L}$ & $\mathrm{H}$ & $\mathrm{L}$ & $\mathrm{L}$ & Pc35 \\
\hline 17 & $\mathrm{~L}$ & $\mathrm{H}$ & $\mathrm{L}$ & $\mathrm{L}$ & $\mathrm{L}$ & - \\
\hline
\end{tabular}

${ }^{\mathrm{a}} \mathrm{H}=$ high infection (virulent reaction); $\mathrm{L}=$ low infection (avirulent reaction). 
fatua, but all were susceptible to five races of $P$. coronata as seedlings, and to common races as adult plants in the field. Brouwer et al. (1982) as well as Burdon et al. (1983) analyzed Australian populations of $A$. fatua and described them as a mixture of resistant and susceptible individuals. Šebesta and Kühn (1990) identified a wide range of crown rust resistance in an A. fatua population collected in Czechoslovakia in 1971. Out of 40 selections, one homozygous line described as A. fatua CS1 was chosen for analysis. Based on the reaction with a single Pc race, the authors stated that its resistance was conditioned by one recessive gene, which was in interaction with one partially dominant gene. The same genotype, A. fatua CS1, was tested by Staletic et al. (2009) using three different races. In this second study, the segregation ratio indicated that resistance was conditioned by a major dominant gene. The current work presents the characteristic of resistance to $P$. coronata in a set of 204 A. fatua accessions coming originally from 29 countries located in North and South America, Asia, Europe, and Africa, and confirms the species potential as a donor of effective resistance genes.

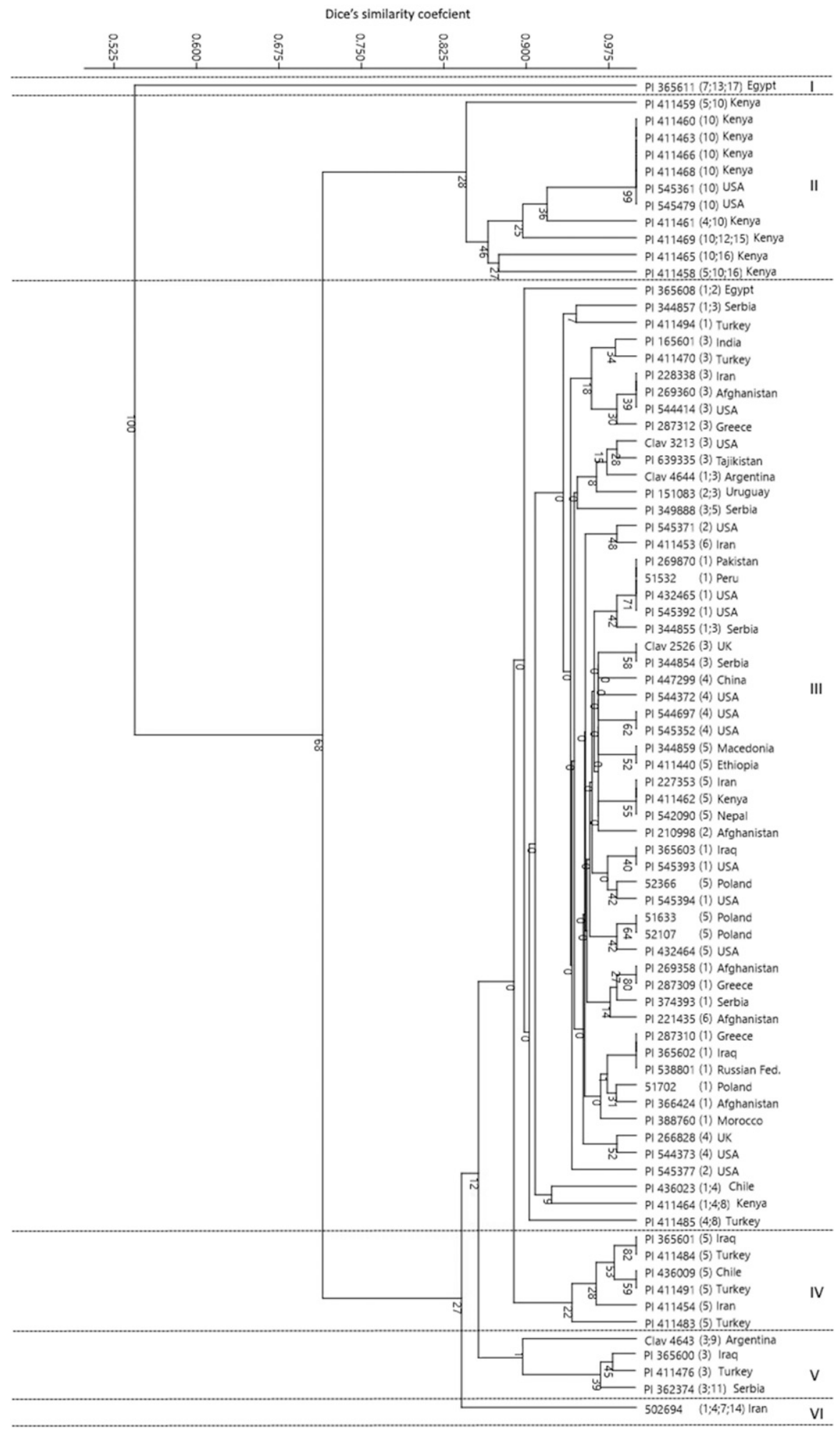

Fig. 2. UPGMA dendrogram of 79 Avena fatua accessions based on the similarity of infection obtained by five Puccinia coronata isolates inoculation. 
Five highly virulent $P$. coronata isolates were used to carry out the host-pathogen test to assess the susceptibility of the studied genotypes. The use of races derived from diverse populations collected in North America and Europe has allowed us to expand the number of defeated genes screened, and in consequence to detect resistance with the greatest potential benefit for current breeding programs. Of studied accessions, $85 \%$ showed a heterogeneous infection pattern, in some cases showing a full range from $\mathrm{S}$ to HR. Of tested genotypes, $61 \%$ could be unambiguously described as susceptible or moderately susceptible to all $P$. coronata races, however. Other studies on wild oat resistance have also revealed variable response to the rust inoculation within single accessions (Carson 2009a, 2010; Sowa et al. 2016; Tan and Carson 2013). Carson (2009a) screened 359 accessions of the tetraploid slender oat, A. barbata, and stated that $39 \%$ were at least moderately resistant to crown rust, some of which displayed variable phenotypes. In a further study encompassing 1,143 A. barbata accessions, $11.4 \%$ of genotypes showed at least a moderately resistant response to $P$. coronata at the seedling and adult plant stage, with only a small number of the tested materials being variable in reaction (Carson 2010). Tan and Carson (2013) in a study involving 332 accessions of 11 different, mainly diploid and tetraploid, wild oat species from Morocco, assessed $49 \%$ of materials as moderately resistant to resistant in a seedling stage and $55 \%$ as moderately resistant to highly resistant in the adult plant stage. A significant proportion of the accessions (25\%) displayed heterogeneous phenotypes. Among the 92 tetraploids, A. magna, A. murphyi, and A. insularis tested by Sowa et al. (2016),
$58.7 \%$ were resistant to at least one crown rust race, and $37 \%$ of accessions were characterized by nonuniform reaction.

The level of phenotype variability within the A. fatua accessions analyzed in this research was significantly higher in comparison with previously studied species, and ranged $85 \%$ of tested genotypes. In case of A. fatua, the tendency to develop populations which are diverse in the infection response may result from the maintenance of assorted set of resistance gene alleles. This phenomenon, called balancing selection, occurs in natural ecosystems, in which natural selection favors different alleles in distinct host populations specialized to specific environments (Möller and Stukenbrock 2017; Mundt and Browning 1985). Genetic variation at the genomic and population levels in both plants and pathogens are the effect of antagonistic interaction between plants and their pathogens (Van der Hoorn et al. 2002). Oates et al. (1983) concluded that in environments where pathogens are widespread and variable, there is a tendency for heterogeneous resistance to arise. The predominance of host genotypes with low or intermediate level of resistance conditioned by various combinations of weak genes or alleles may be beneficial for the host population in a long-term perspective.

Crown rust, as the most widespread and harmful oat disease, appears in almost every region of oat cultivation (Chong 2003). Loskutov (2007) claims that the most resistant genotypes should be expected in the center of origin of the genus Avena, believed to be located in the Mediterranean region. This thesis works in the case of $P c$ genes derived from animated oat A. sterilis (Gnanesh et al.

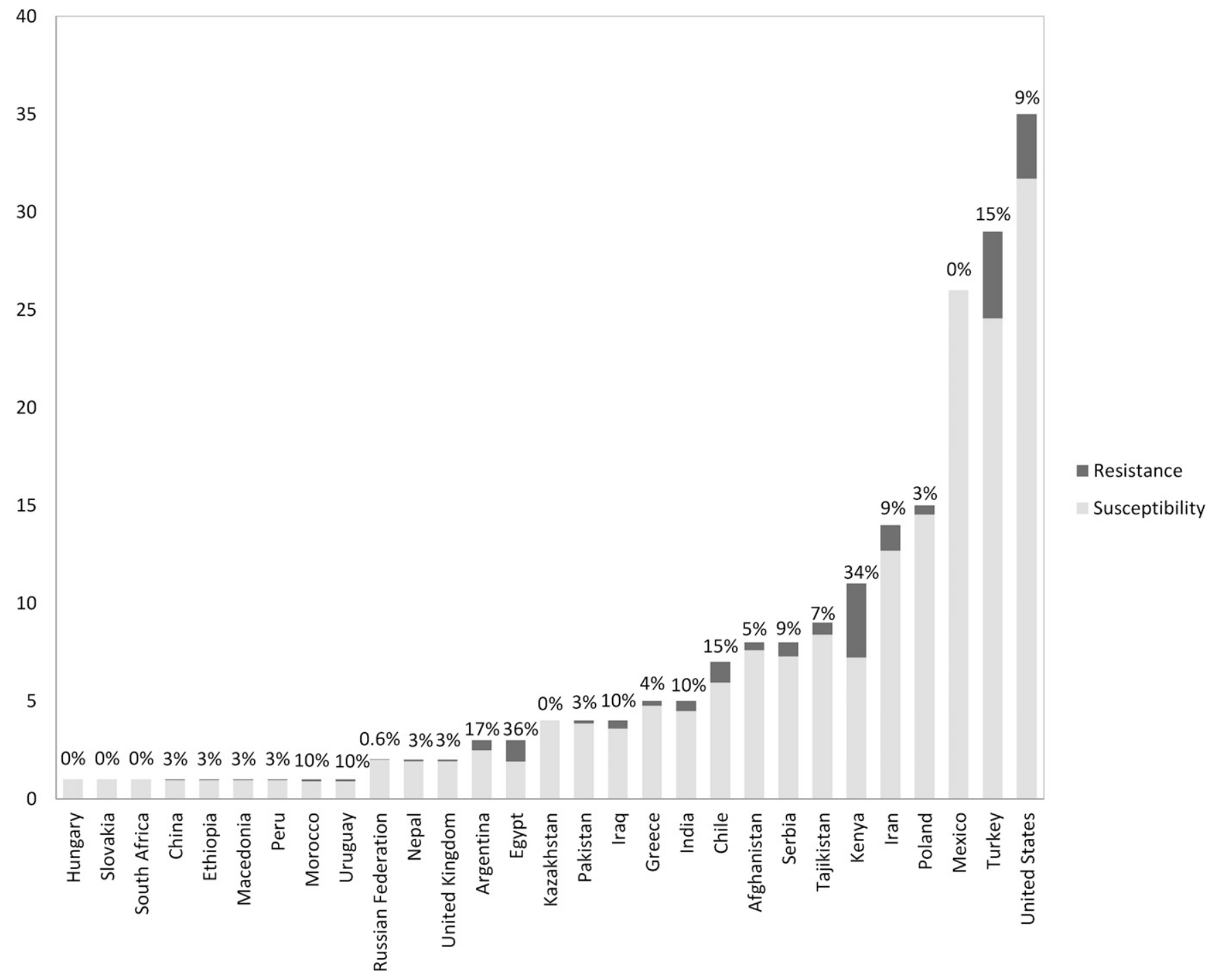

Fig. 3. Percentage of resistance reaction within Avena fatua accessions from different countries to crown rust isolates used in host-pathogen tests. 
2014; Šebesta et al. 2003). A. fatua is one of the most common and obnoxious cereal weeds worldwide, and so may be subjected to different disease dynamics (Briggle and Youngs 1975; Holm et al. 1991). In our research, A. fatua genotypes from a given country were represented proportionally to their availability in the gene banks. The distribution of resistant accessions varied and was not concentrated in one particular area. The largest number of accessions tested was from the United States (36), and as many as 15 exhibited resistance. However, this resistance was mostly on a very low level, occurring in single seedlings within accessions. Only in the cases of PI 545361 from Texas and PI 545479 from Oklahoma with IP 10, every screened seedling was found to be resistant to 94(63) and CR257. This phenotype corresponded to reference line Pc14 and was also observed in 9 highly resistant accessions from Kenya, which composed the second cluster of the UPGMA dendrogram. IP 10 was the most common profile for Kenyan accessions, where each of the 11 screened genotypes showed exceptionally high and homogenous levels of resistance. Genotype 502694 from Iran and Egyptian accession PI 365611, characterized by distinctive and complex profiles of resistance, were strikingly different from the remaining accessions. Within the first mentioned genotype, four of six seedlings were resistant, each showing different IP based on the resistance to three crown rust pathotypes: 51(22), CR230, and CR241. Similar variability was observed in PI 365611. In this case, all six tested seedlings displayed variable resistance to two to four $P$. coronata pathotypes (51U(22), CR230, CR241, and CR257). Within this accession, two plants were resistant to all four crown rust races (IP 17) and presented the highest level of resistance of all tested materials. Overall, five profiles of infection corresponding to resistance to three of five analyzed crown rust pathotypes were identified. Two of them, PI 12, indicating resistance to 51(22); 94(63) and CR257 as well as PI 15 (94(63); CR230, and CR257) were unique and appeared within accession PI 411469 from Kenya. Overall, 12 of 18 determined infection profiles did not correspond to the phenotype of any of the reference Pc lines used in the research. This suggests they result from the presence of the new sources of resistance to crown rust, which in the cases of PI 365611 and PI 411469 should be highly effective against current pathogens.

Currently, one of the major challenges of the oat breeding programs is the durability of genetic resistance. New genes identified in the study may be valuable as components in the resistance gene pyramids, even where their levels of resistance are equivalent to existing $P c$ genes. Pyramiding is considered one of the most promising strategies of plant resistance breeding (Joshi and Nayak 2010). In recent years, quantitative resistance has also gained a great interest, and due to the development of molecular biology, improved tools are becoming available to better integrate this type of resistance into breeding strategies (PiletNayel et al. 2017). Combinations of partial resistance are expected to result in much more durable polygenic resistance than can be obtained through use of single major genes. Future work will clarify the genetic basis of the resistances observed here, as well as confirm their potential utility in the field, where resistance may be affected by the plant development stage as well as other factors such as temperature (Niks et al. 2015). There remains considerable scope to investigate $A$. fatua as a model for selection of durable disease resistance under agricultural conditions.

\section{Acknowledgments}

We thank Dr. J. Menzies (Agriculture and Agri-Food Canada, Morden, MB, Canada) for providing crown rusts isolates.

\section{Literature Cited}

Adamczewski, K., Kierzek, R., and Matysiak, K. 2013. Wild oat (Avena fatua L.) biotypes resistant to acetolactate synthase and acetyl-CoA carboxylase inhibitors in Poland. Plant Soil Environ. 59:432-437.

Aung, T., Chong, J., and Leggett, M. 1996. The transfer of crown rust resistance Pc94 from a wild diploid to cultivated hexaploid oat. Pages 167-171 in: 9th International European and Mediterranean Cereal Rust and Powdery Mildews Conference 2-6 September 1996. G. H. J. Kema, R. E. Niks, and R. A. Daamen, eds. Wageningen, European and Mediterranean Cereal Rust Foundation, Lunteren, Netherlands.

Aung, T., Zwer, P., Park, R., Davies, P., Sidhu, P., and Dundas, I. 2010. Hybrids of Avena sativa with two diploid wild oats (CIav6956) and (CIav7233) resistant to crown rust. Euphytica 174:189-198.
Briggle, L. W. 1974. Development of useful germplasm from wild oat species. Oat Newsl. 24:33-34.

Briggle, L. W., and Youngs, V. I. 1975. Collection and evaluation of Avena fatua L. Oat Newsl. 25:9-10.

Brouwer, J. B., Oats, J. D., and Burdon, J. J. 1982. Variability of Puccinia coronata avenae in Australia. Oat Newsl. 32:32-33.

Burdon, J. J., Oates, J. D., and Marshall, D. R. 1983. Interactions between Avena and Puccinia species. I. The wild hosts: Avena barbata Pott Ex Link, A. fatua L., A. ludoviciana Durieu. J. Appl. Ecol. 20:571-584.

Cabral, A. L., Gnanesh, B. N., Fetch, J. M., McCartney, C., Fetch, T., Park, R. F., Menzies, J. G., McCallum, B., Nanaiah, G. K., and Goyal, A. 2014. Oat fungal diseases and the application of molecular marker technology for their control. Pages 343-358 in: Future Challenges in Crop Protection Against Fungal Pathogens. A. Goyal and C. Manoharachary, eds. Springer Science+Business Media, New York.

Cabral, A. L., and Park, R. F. 2014. Seedling resistance to Puccinia coronata f. sp avenae in Avena strigosa, A. barbata and A. sativa. Euphytica 196:385-395.

Cabral, A. L., Singh, D., and Park, R. F. 2011. Identification and genetic characterisation of adult plant resistance to crown rust in diploid and tetraploid accessions of Avena. Ann. Appl. Biol. 159:220-228.

Carson, M. L. 2009a. Broad-spectrum resistance to crown rust, Puccinia coronata f. sp. avenae, in accessions of the tetraploid slender oat, Avena barbata. Plant Dis. 93:363-366.

Carson, M. L. 2009b. Crown rust development and selection for virulence in Puccinia coronata f. sp. avenae in an oat multiline cultivar. Plant Dis. 93: 347-353.

Carson, M. L. 2010. Additional sources of broad-spectrum resistance to Puccinia coronata f. sp. avenae from Canadian accessions of Avena barbata. Plant Dis. 94:1405-1410.

Carson, M. L. 2011. Virulence in oat crown rust (Puccinia coronata f. sp. avenae) in the United States from 2006 through 2009. Plant Dis. 95:1528-1534.

CDL. 2017. Cereal Disease Laboratory, St. Paul, Minnesota. https://www.ars.usda. gov/midwest-area/stpaul/cereal-disease-lab/docs/resistance-genes/resistancegenes/.

Chaves, M. S., Martinelli, J. A., Wesp, C. de L., and Graichen, F. A. S. 2008. The cereal rusts: An overview. Pest Technol. 2:38-55.

Chong, J. 2003. Disease of Oat. Pages 74-88 in: Diseases of Field Crops in Canada K. Bailey, B. Gossen, R. Gugel, and R. Morrall, eds. CPS Press, Saskatoon.

Chong, J., Gruenke, J., Dueck, R., Mayert, W., Mitchell Fetch, J. W., and McCartney, C. A. 2011. Virulence of Puccinia coronata $\mathrm{f}$. sp. avenae in the eastern prairie region of Canada during 2007-2009. Can. J. Plant Pathol. 33: 77-87.

Chong, J., Leonard, K. J., and Salmeron, J. J. 2000. A North American system of nomenclature for Puccinia coronata f. sp. avenae. Plant Dis. 84:580-585.

Dice, L. R. 1945. Measures of the amount of ecologic association between species. Ecology 26:297-302.

Dyck, P. L., and Zillinsky, F. J. 1963. Inheritance of crown rust resistance transferred from diploid to hexaploid oats. Can. J. Genet. Cytol. 5:398-407.

Fetch, T., McCallum, B., Menzies, J., Rashid, K., and Tenuta, A. 2011. Rust diseases in Canada. Prairie Soils and Crops 4:86-96.

Gnanesh, B. N., Fetch, J. M., Zegeye, T., McCartney, C. A., and Fetch, T. 2014 Oat. Pages 51-73 in: Alien Gene Transfer in Crop Plants. A. Pratap and J. Kumar, eds. Springer, New York.

Gnanesh, B. N., Mitchell Fetch, J. W., Menzies, J. G., Beattie, A. D., Eckstein, P. E., and McCartney, C. A. 2013. Chromosome location and allele-specific PCR markers for marker-assisted selection of the oat crown rust resistance gene $P c 91$. Mol. Breed. 32:679-686.

Hammer, Ø., Harper, D. A. T., and Ryan, P. D. 2001. PAST: Paleontological Statistics Software Package for education and data analysis. Palaeontol. Electronica 4:1-9.

Holm, L. G., Plucknett, D. L., Pancho, J. V., and Herberger, J. P. 1991. The world's worst weeds. Distribution and biology. The University Press of Hawaii, Honolulu.

Hsam, S. L. K., Peters, N., Paderina, E. V., Felsenstein, F., Oppitz, K., and Zeller, F. J. 1997. Genetic studies of powdery mildew resistance in common oat (Avena sativa L.) I. Cultivars and breeding lines grown in Western Europe and North America. Euphytica 96:421-427.

Jellen, E., and Leggett, J. M. 2006. Cytogenetic Manipulation in Oat Improvement. Pages 199-231 in: Genetic Resources, Chromosome Engineering, and Crop Improvement, Genetic Resources Chromosome0 Engineering \& Crop Improvement. R. J. Singh, and P. P. Jauhar, eds. CRC Press, Boca Raton.

Jiráková, H., and Hanzalová, A. 2008. Crown rust pathotypes determined on oats in the Czech Republic from 2004 to 2006 and reaction to oat cultivars. Czech J. Genet. Plant Breed. 44:60-65.

Joshi, R. K., and Nayak, S. 2010. Gene pyramiding-A broad spectrum technique for developing durable stress resistance in crops. Biotechnol. Mol. Biol. Rev. 5:51-60.

Loskutov, I. G. 2007. On evolutionary pathways of Avena species. Genet. Resour Crop Evol. 55:211-220.

Loskutov, I. G., and Rines, H. W. 2011. Avena. Pages 109-183 in: Wild Crop Relatives: Genomic and Breeding Resources. C. Kole, ed. Springer Berlin Heidelberg, Berlin, Heidelberg. 
Menzies, J. G., Xue, A., Dueck, R., and Greunke, J. 2015. Virulence of Puccinia coronata f. sp. avenae in Canada; 2010 to 2014. Page 95 in: 14th International Cereal Rust and Powdery Mildew Conference 5-8 July 2015. Copenhagen, Denmark.

Mitchell Fetch, J. W., Duguid, S. D., Brown, P. D., Chong, J., Fetch, T. G., Haber, S. M., Menzies, J. G., Ames, N., Noll, J., Aung, T., and Stadnyk, K. D. 2007. Leggett oat. Can. J. Plant Sci. 87:509-512.

Möller, M., and Stukenbrock, E. H. 2017. Evolution and genome architecture in fungal plant pathogens. Nat. Rev. Microbiol. 15:756-771.

Mundt, C. C., and Browning, J. A. 1985. Genetic diversity and cereal rust management. Pages 527-560 in: The Cereal Rusts. W. R. Bushnell, and A. P. Roelfs, eds. Vol. 2. Academic Press, Orlando, Florida.

Nazareno, E. S., Li, F., Smith, M., Park, R. F., Kianian, S. F., and Figueroa, M. 2017. Puccinia coronata f. sp. avenae: A threat to global oat production. Mol. Plant Pathol. 19:1-14.

Niks, R. E., Qi, X., and Marcel, T. C. 2015. Quantitative resistance to biotrophic filamentous plant pathogens: Concepts, misconceptions, and mechanisms. Annu. Rev. Phytopathol. 53:445-470.

Oates, J. D., Burdon, J. J., and Brouwer, J. B. 1983. Interactions between Avena and Puccinia species. II. The pathogens: Puccinia coronata $\mathrm{Cda}$ and P. graminis Pers. f. sp. avenae Eriks. \&. Henn. J. Appl. Ecol. 20:585-596.

Owen, M. J., and Powles, S. B. 2009. Distribution and frequency of herbicideresistant wild oat (Avena spp.) across the Western Australian grain belt. Crop Pasture Sci. 60:25-31.

Pilet-Nayel, M.-L., Moury, B., Caffier, V., Montarry, J., Kerlan, M.-C., Fournet, S., Durel, C.-E., and Delourme, R. 2017. Quantitative resistance to plant pathogens in pyramiding strategies for durable crop protection. Front. Plant Sci. 8:1838.

Rines, H. W., Miller, M. E., Carson, M., Chao, S., Tiede, T., Wiersma, J., and Kianian, S. F. 2018. Identification, introgression, and molecular marker genetic analysis and selection of a highly effective novel oat crown rust resistance from diploid oat, Avena strigosa. Theor. Appl. Genet. 131:721-733.
Šebesta, J., and Kühn, F. 1990. Avena fatua L. subsp. fatua v. glabrata Peterm. subv. pseudo-basifixa Thell. as a source of crown rust resistance genes. Euphytica 50:51-55.

Šebesta, J., Zwatz, B., Roderick, H., Corazza, L., Manisterski, J., and Stojanovic, S. 2003. Incidence of crown rust and virulence of Puccinia coronata cda. f.sp. avenae eriks. and the effectiveness of $P c$ genes for resistance in Europe, Middle East and North Africa. Arch. Phytopathol. Plant Prot. 36:179-194.

Simons, M. D., and Briggle, L. W. 1984. Screening for tolerance to Puccinia coronata in progenies of visually susceptible strains of Avena fatua. Phytopathology 74:1271.

Simons, M. D., Martens, J. W., McKenzie, R. I. H., Nishiyama, I., Sadanaga, K., Sebesta, J., and Thomas, H. 1978. Oats: A Standardized System of Nomenclature for Genes and Chromosomes and Catalog of Genes Governing Characters. Science and Education Administration, U.S. Dep. Agric. Washington, D.C.: Science and Education Administration, and Iowa Agricultural and Home Economics Experiment Station.

Sowa, S., Paczos-Grzęda, E., Koroluk, A., Okoń, S., Ostrowska, A., Ociepa, T. Chrząstek, M., and Kowalczyk, K. 2016. Resistance to Puccinia coronata f. sp. avenae in Avena magna, A. murphyi, and A. insularis. Plant Dis. 100: 1184-1191

Staletic, M., Milovanovic, M., Djekiš, V., and Markovic, A. 2009. The efficiency of $P c$ resistance genes derived from Avena sterilis against Puccinia coronata avenae. Pages 390-394 in: Genetics, Plant Breeding and Seed Production. 44th Croatian and 4th International Symposium on Agriculture, Opatija.

Tan, M. Y. A., and Carson, M. L. 2013. Screening wild oat accessions from Morocco for resistance to Puccinia coronata. Plant Dis. 97:1544-1548.

Thill, D. C., O'Donovan, J. T., and Mallory-Smith, C. A. 1994. Integrated weed management strategies for delaying herbicide resistance in wild oats. Phytoprotection 75:61-70

Van der Hoorn, R. A., De Wit, P. J., and Joosten, M. H. 2002. Balancing selection favors guarding resistance proteins. Trends Plant Sci. 7:67-71. 